\title{
Latent structure of the proposed ICD-11 post-traumatic stress disorder symptoms: implications for the diagnostic algorithm
}

David Forbes, Emma Lockwood, Mark Creamer, Richard A. Bryant, Alexander C. McFarlane, Derrick Silove, Angela Nickerson and Meaghan O'Donnell

\section{Background}

The latent structure of the proposed ICD-11 post-traumatic stress disorder (PTSD) symptoms has not been explored.

\section{Aims \\ To investigate the latent structure of the proposed ICD-11 PTSD symptoms}

\section{Method}

Confirmatory factor analyses using data from structured clinical interviews administered to injury patients $(n=613)$ 6 years post-trauma. Measures of disability and psychological quality of life (QOL) were also administered.

\section{Results}

Although the three-factor model implied by the ICD-11 diagnostic criteria fit the data well, a two-factor model provided equivalent, if not superior, fit. Whereas diagnostic criteria based on this two-factor model resulted in an increase in PTSD point prevalence $(5.1 \%$ v. $3.4 \% ; z=2.32$, $P<0.05)$, they identified individuals with similar levels of disability $(P=0.933)$ and QOL $(P=0.591)$ to those identified by the ICD-11 criteria.

\section{Conclusions}

Consistent with theorised reciprocal relationships between re-experiencing and avoidance in PTSD, these findings support an alternative diagnostic algorithm requiring at least two of any of the four re-experiencing/avoidance symptoms and at least one of the two hyperarousal symptoms.

\section{Declaration of interest}

R.A.B. served on the DSM-5 PTSD/Trauma/Dissociative Work Group.
The ICD-11 is due for publication by the World Health Organization in 2015. To enhance clinical utility and ease of application across a range of contexts, the proposed diagnostic criteria for ICD-11 post-traumatic stress disorder (PTSD) comprise just six symptoms. ${ }^{1}$ In addition to exposure to a traumatic event, the proposed ICD-11 tripartite diagnostic algorithm requires at least one of two re-experiencing symptoms (nightmares or flashbacks), at least one of two avoidance symptoms (avoidance of thoughts or memories associated with the event or avoidance of people, places, conversations, activities or situations associated with the event) and at least one of two hyperarousal symptoms (hypervigilance and exaggerated startle response). ${ }^{2}$ Thus, the proposed criteria presume a three-factor model of the latent structure of ICD-11 PTSD symptoms, with factors corresponding to re-experiencing, avoidance and hyperarousal clusters, and with two symptoms specified to load on each factor. However, whether such a model best reflects the latent structure of ICD-11 PTSD is an empirical question that can be addressed using confirmatory factor analysis (CFA). ${ }^{3}$

These proposed ICD-11 diagnostic criteria for PTSD stand in contrast to the criteria for PTSD outlined in DSM- $5,{ }^{4}$ which require at least one of five intrusion symptoms, at least one of two avoidance symptoms, at least two of seven negative alterations in cognitions and mood symptoms and at least one of six arousal and reactivity symptoms. The ICD-11 avoidance symptoms directly map on to the DSM-5 avoidance symptoms, whereas the ICD-11 re-experiencing and hyperarousal symptoms are subsets of the DSM-5 intrusion and arousal and reactivity symptoms. respectively. Notably, studies of the latent structure of DSM-5 PTSD symptoms ${ }^{4-6}$ have found evidence for very high correlations $(<0.90)$ and hence poor discriminant validity ${ }^{7,8}$ between its re-experiencing and avoidance factors. Thus, this study assesses and compares the goodness-of-fit of the presumed three-factor model of ICD-11 PTSD symptoms and a two-factor model encompassing a combined re-experiencing/avoidance factor and a hyperarousal factor. The study also assesses the impact of a diagnostic algorithm based on each of these models on the point prevalence of PTSD and accompanying levels of comorbidity, disability and psychological quality of life (QoL). Determining the capacity of the proposed criteria and diagnostic algorithm to perform adequately on these parameters is essential if we are to have faith in the proposed PTSD diagnosis in describing and differentiating patients who experience the condition following trauma.

\section{Method}

\section{Participants}

Participants comprised 613 traumatic injury survivors who were weekday admissions to specialised trauma services at one of four hospitals in three Australian states between April 2004 and February 2006. Data obtained at 6-year follow-up after their initial recruitment were used in this study. Participants were recruited as part of a prospective cohort study investigating the psychiatric sequelae of traumatic injury. ${ }^{9}$ Participants were randomly selected from a larger pool of injury patients using an automated, random selection procedure, stratified by length of stay. Participants were considered for inclusion in the study if they had experienced a traumatic injury requiring hospital admission of greater than $24 \mathrm{~h}$, had either no brain injury or a mild traumatic brain injury, ${ }^{10}$ were aged between 16 and 70 years, and had sufficient English comprehension to complete the assessment. Patients were excluded from the study if they were suicidal or had psychosis. Written informed consent was obtained from participants after a complete description of the study was provided. The research 
was approved by the Human Research and Ethics Committees in each hospital.

A total of 1590 people were initially approached for inclusion in this study in 2004-2006. Of these, 1138 (72\%) consented to participate, and $613(39 \%)$ were available for follow-up after 6 years. Of these 613 , the majority were male $(72 \% ; n=440)$ and the average age was 40 years at the time of their admission to hospital (s.d.=13.4). Individuals who refused to participate in the study or were unavailable for follow-up at 6 years did not differ from participants in gender, the presence of a mild traumatic brain injury, education, mechanism of injury, length of stay or Injury Severity Score (ISS). ${ }^{11}$ However, participants were more likely to have been older at the time of admission than those who were unavailable for follow-up at 6 years (40 v. 36 years; $t=-4.4, P<0.001)$. The 613 participants spent on average 13 days (s.d.=14.2) in hospital, with $43 \%$ experiencing a mild traumatic brain injury as a result of their injury and $16 \%$ requiring intensive care unit admission. The principal mechanism of injury was motor vehicle accidents $(n=405,66 \%)$; other mechanisms of injury included falls $(n=99,16 \%)$, assaults $(n=36,6 \%)$, workplace injuries $(n=29,5 \%)$ and other injuries $(n=44,7 \%)$. The mean ISS was 11 (s.d. $=7.7)$, which is in the moderate range of severity.

\section{Measures}

\section{PTSD symptoms}

The Clinician Administered PTSD Scale (CAPS) ${ }^{12,13}$ was used to assess current PTSD symptoms at 6 years post-injury. Although this structured clinical interview was developed to assess the DSM-IV ${ }^{14}$ rather than the ICD diagnosis of PTSD, the CAPS includes two items that approximately index each of the proposed six ICD-11 symptoms. These items measure the frequency and intensity of the symptoms on five-point $(0-4)$ ordinal scales. The CAPS was administered via telephone by research assistants who had each attained at least a 4-year bachelor's degree in psychology and who had been trained in its administration. To test interrater reliability, $5 \%$ of all CAPS interviews were selected using a random number generator program and assessed by an independent assessor who was masked to the original scoring. Overall, the diagnostic consistency on the CAPS was 100\%.

\section{Comorbidity}

The Mini International Neuropsychiatric Interview Version 5.5 $(\mathrm{MINI})^{15}$ was used to assess current diagnoses of major depressive episode, generalised anxiety disorder, panic disorder and travel phobia. The MINI is a short, structured diagnostic interview based on the DSM-IV and ICD-10 ${ }^{16}$ classifications.

\section{Disability}

The 12-item World Health Organization Disability Assessment

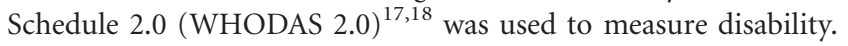
Each item on the WHODAS 2.0 is rated on a five-point $(0-4)$ scale, and items are summed ${ }^{19}$ to create a total disability score ranging from 0 (no disability) to 48 (complete disability). Disability was treated as both a continuous and a binary variable in this study. Although there is no agreed upon cut-point for identifying people with significant disability, people scoring $10-48$ are in the top $10 \%$ of the population distribution of WHODAS 2.0 scores and are likely to have clinically significant disability. ${ }^{19}$ Thus, a score of $>9$ was used as the threshold for high disability $v$. low disability.

\section{QOL}

The eight-item psychological domain subscale from the World Health Organization Quality of Life - Bref (WHOQOL-bref) ${ }^{20}$ was used to measure of QoL. In the current study, a scoring algorithm was used to standardise scores to a $0-100 \mathrm{scale}^{21}$ with higher scores indicating higher QoL. We also treated QoL as both a continuous and a binary variable in this study. Based on Australian population norms, a score of less than 55.5 was used as the cut-off for poor psychological QoL. ${ }^{22}$

\section{Data analysis}

The CAPS symptom data from the 613 participants were subject to CFAs using Mplus version 7.11 for Windows $7 .{ }^{23}$ Three-, two- and one-factor models of the proposed ICD-11 PTSD symptoms were tested for goodness of fit. In the first instance, the fit of the three-factor model implied by the proposed ICD-11 diagnostic criteria was tested. In this model items measuring DSM-IV symptoms B2 (nightmares) and B3 (flashbacks) were specified to load on a 're-experiencing' factor, items measuring symptoms $\mathrm{C} 1$ (avoidance of trauma-related thoughts and feelings) and C2 (avoidance of trauma-related people and places) onto an 'avoidance' factor and items measuring symptoms D4 (hypervigilance) and D5 (exaggerated startle response) onto a 'hyperarousal' factor. Next, the fit of a two-factor model was tested in which items assessing symptoms B2, B3, C1 and C2 were specified to load onto a combined 're-experiencing/avoidance' factor and items assessing D4 and D5 were specified to load onto a 'hyperarousal' factor. Finally, the fit of a one-factor model was tested in which items assessing all six ICD-11 symptoms were specified to load onto a single factor.

As the items measuring each symptom are ordinal, mean- and variance-adjusted weighted least squares estimation (WLSMV) was first used to obtain parameter estimates and indices of model fit. ${ }^{7,24,25}$ To handle missing data, the default option for categorical outcomes using weighted least squares estimation was used, which allows missingness to be a function of the observed covariates but not the observed outcomes. When there are no covariates in the model, this is analogous to pair-wise present analysis. ${ }^{26}$ As the frequency and intensity items for each symptom were allowed to load onto their specified factors separately, some residual covariation or method variance was expected. Thus, the residual covariances for each pair of frequency and intensity items were estimated and tested for significance. ${ }^{7,27-29}$

Goodness of fit was evaluated using the WLSMV- $\chi^{2}$ statistic and other fit indices in line with the two-index strategy of $\mathrm{Hu} \&$ Bentler. ${ }^{30}$ This strategy places most emphasis on the root mean squared error of approximation (RMSEA) ${ }^{31}$ together with the Comparative Fit Index $(\mathrm{CFI})^{32}$ and the Tucker-Lewis Index (TLI), ${ }^{33}$ as $\chi^{2}$ may be an overly sensitive test of fit in large samples. ${ }^{8,34}$ An RMSEA of less than 0.06 and CFI or TLI greater than 0.95 were regarded as indicating good fit, and an RMSEA of less than 0.08 , adequate fit. ${ }^{30}$ The fit of models was directly compared using $\chi^{2}$ difference testing, supplemented with $90 \%$ confidence intervals for RMSEA values. The analyses were then re-run using maximum likelihood estimation instead of WLSMV, to enable further direct comparison of goodness of fit using the Bayesian Information Criterion (BIC). As the item for each symptom was non-normally distributed (Kolmogorov-Smirnov and Shapiro-Wilk tests $P<0.001$ for all items), robust maximum likelihood estimation was used, as it is able to handle nonnormally distributed items as well as ordinal items with at least five response categories. ${ }^{7,25}$ A ten-point BIC difference represents a 150:1 likelihood that the model with the lower BIC value fits best; a difference in the 6-10 point range indicates 'strong' support, and a difference greater than 10 indicates 'very strong' support. $^{35}$

If a one- or two- factor model was found to provide the best fit to the data, alternative diagnostic criteria based on that model 
were formulated, so the point prevalence of PTSD derived using the alternative criteria could be compared with that derived using the three-factor ICD-11 criteria. As all individuals meeting the three-factor criteria would also meet one- or two-factor diagnostic criteria, disability and psychological QoL scores and rates of comorbid mood and anxiety disorders in those who met the three-factor criteria were compared with scores and rates among those who met the less restrictive criteria only. Disability and psychological QoL scores for these groups were also compared with those of individuals who did not qualify for a diagnosis using either set of criteria. This was in order to determine whether a more inclusive set of diagnostic criteria (i.e. that based on a one- or two-factor model) (a) identified individuals experiencing levels of disability and psychological QoL equivalent to those identified using the ICD-11 criteria, and (b) were not merely identifying individuals for whom any higher disability and lowered QoL scores might be attributable to higher rates of comorbid conditions. Comparisons were made using $z$-scores (point prevalence rates) and one-way analyses of variance (ANOVAs) (continuous disability and psychological QoL scores).

\section{Results}

As previously reported by O'Donnell et al ${ }^{36}$ the point prevalence of ICD-11 PTSD in the sample was 3.4\% (21 individuals out
613). Fit indices for each of the specified ICD-11 models are presented in Table 1. All models demonstrated good fit according to RMSEA, CFI and TLI estimated using WLSMV. Although the CFI and TLI indicated a high level of fit for each model, the three-factor model demonstrated superior fit to the one-factor model using WLSMV- $\chi^{2}$ difference testing. Further, BICs derived using robust maximum likelihood estimation provided 'very strong' support for the three-factor model over the one-factor model. Of note, however, and consistent with previous research, the correlation between the re-experiencing factor (symptoms $\mathrm{B} 2$ and B3) and the avoidance factor (C1 and $\mathrm{C} 2)$ in the threefactor model was 0.94 with a standard error of 0.07 (Table 2), indicating a potential lack of discriminant validity for these two factors. The CFI and TLI for the alternative two-factor model in which items assessing symptoms B2, B3, C1 and C2 were specified to load onto a first 're-experiencing/avoidance' factor and items assessing D4 and D5 were specified to load onto a second 'hyperarousal' factor indicated a high level of fit comparable with that of the one- and three-factor models. Notably, the RMSEA for the two-factor model fell below the $90 \%$ confidence interval for the one-factor model, suggesting a superior level of fit for the two-factor model, and $\chi^{2}$ difference testing also indicated a superior fit for the two-factor compared with the one-factor model. Comparing the two- and three-factor models, RMSEAs for each model fell within the RMSEA confidence intervals for the other and $\chi^{2}$ difference testing failed to find superior fit for

\begin{tabular}{|c|c|c|c|c|c|c|c|c|c|c|}
\hline \multirow[b]{3}{*}{ Model $^{a}$} & \multirow[b]{3}{*}{$\chi^{2 b}$} & \multirow[b]{3}{*}{ d.f. ${ }^{b}$} & \multirow[b]{3}{*}{$\mathrm{CF}^{\mathrm{b}}$} & \multirow[b]{3}{*}{$T L^{b}$} & \multirow[b]{3}{*}{$\operatorname{RMSEA}^{\mathrm{b}}(90 \% \mathrm{Cl})$} & \multicolumn{4}{|c|}{$\chi^{2}$ difference testing ${ }^{\mathrm{b}}$} & \multirow[b]{3}{*}{$\mathrm{BIC}^{\mathrm{C}}$} \\
\hline & & & & & & \multicolumn{2}{|c|}{ v. 1-factor model } & \multicolumn{2}{|c|}{ v. 2-factor model } & \\
\hline & & & & & & $\chi^{2}$ & $P$ & $\chi^{2}$ & $P$ & \\
\hline 3-factor ICD-11 model & $67.323^{*}$ & 45 & 0.999 & 0.998 & $0.028(0.012-0.042)$ & $13.374^{*}$ & 0.004 & 2.221 & 0.329 & 11433.3 \\
\hline 2-factor model & $65.249^{*}$ & 47 & 0.999 & 0.998 & $0.025(0.006-0.039)$ & $10.288^{*}$ & 0.001 & & & 11426.6 \\
\hline 1-factor model & $90.103^{*}$ & 48 & 0.997 & 0.996 & $0.038(0.026-0.050)$ & & & & & 11450.3 \\
\hline
\end{tabular}

Table 2 Factor loadings and correlations (standard errors) for the three-factor ICD-11 model and alternative two-factor model

\begin{tabular}{|c|c|c|c|c|c|}
\hline & \multicolumn{3}{|c|}{ Three-factor ICD-11 model } & \multicolumn{2}{|c|}{ Two-factor model } \\
\hline & Re-experiencing & Avoidance & Hyperarousal & Re-experiencing/avoidance & Hyperarousa \\
\hline \multicolumn{6}{|l|}{ Loadings } \\
\hline \multicolumn{6}{|c|}{ B2 Distressing dreams } \\
\hline Frequency & $0.81(0.07)$ & & & $0.76(0.05)$ & \\
\hline Intensity & $0.80(0.07)$ & & & $0.75(0.05)$ & \\
\hline \multicolumn{6}{|l|}{ B3 Flashbacks } \\
\hline Frequency & $0.67(0.07)$ & & & $0.64(0.06)$ & \\
\hline Intensity & $0.64(0.07)$ & & & $0.61(0.06)$ & \\
\hline \multicolumn{6}{|c|}{ C1 Avoidance of memories } \\
\hline Frequency & & $0.84(0.04)$ & & $0.85(0.04)$ & \\
\hline Intensity & & $0.85(0.04$ & & $0.85(0.04)$ & \\
\hline \multicolumn{6}{|c|}{ C2 Avoidance of external reminders } \\
\hline Frequency & & $0.75(0.04)$ & & $0.75(0.05)$ & \\
\hline Intensity & & $0.81(0.04)$ & & $0.81(0.04)$ & \\
\hline \multicolumn{6}{|c|}{ D4 Hypervigilance } \\
\hline Frequency & & & $0.77(0.05)$ & & $0.77(0.05)$ \\
\hline Intensity & & & $0.81(0.05)$ & & $0.81(0.05)$ \\
\hline \multicolumn{6}{|c|}{ D5 Exaggerated startle response } \\
\hline Frequency & & & $0.72(0.05)$ & & $0.72(0.05)$ \\
\hline Intensity & & & $0.75(0.05)$ & & $0.75(0.05)$ \\
\hline \multicolumn{6}{|c|}{ Factor correlations } \\
\hline Avoidance & $0.94(0.07)$ & & & & \\
\hline Hyperarousal & $0.74(0.09)$ & $0.84(0.06)$ & & $0.82(0.06)$ & \\
\hline
\end{tabular}


the three-factor model, despite its fewer degrees of freedom. The BICs derived using robust maximum likelihood estimation provided 'very strong' support for the two-factor model over the one-factor model, as well as 'strong' support for the two-factor model over the three-factor model (difference of 6.7).

Thus, given the high correlations between the re-experiencing and avoidance factors of the three-factor model, its lack of superior fit relative to the alternative two-factor model and the strong support for the two-factor over the three-factor model from the BIC, the two-factor model was considered a more parsimonious, and thus potentially preferred, alternative to the three-factor model. Factor loadings for both the two- and three-factor models are presented in Table 2. Diagnostic criteria based on this model (at least two of B2, B3, C1 or C2, and at least one of D4 or D5) produced an alternative point prevalence of $5.1 \%$ (31 individuals out of 613 ), which was significantly higher than the $3.4 \%$ point prevalence produced by the three-factor ICD-11 diagnostic criteria $(z=2.32, P<0.05)$.

Disability (WHODAS 2.0) and psychological QoL (WHOQOLbref psychological domain) data were available for 540 and 541 participants respectively. $T$-tests indicated no difference in ICD-11 PTSD symptom severity (sum of CAPS item scores for the six ICD-11 symptoms) between individuals for whom WHODAS 2.0 and WHOQOL-bref was and was not available (disability: $t=1.81, \quad$ d.f. $=611, \quad P=0.07 ; \quad$ psychological QoL: $t=1.89$, d.f. $=611, P=0.06$ ). Table 3 presents the average disability and psychological QoL scores for individuals meeting both the twoand three-factor criteria for PTSD, individuals meeting only the two-factor criteria for PTSD, and individuals meeting neither set of criteria. The average disability scores for individuals qualifying for a diagnosis of PTSD based on either set of criteria fell in the high range, whereas the average disability score for individuals who did meet criteria for PTSD using either set did not fall in the high range. Similarly, the average psychological QoL score for individuals qualifying for a diagnosis of PTSD using either set of criteria fell in the low range, whereas the average psychological QoL score for individuals who did meet criteria for PTSD using either set did not. One-way ANOVAs indicated that individuals qualifying for a diagnosis of PTSD using either the two- and three-factor criteria had significantly higher disability and lower psychological QoL scores than individuals not qualifying for a diagnosis using either criteria (disability: $F(2,537)=24.0, P<0.001$; Tukey's Honest Significant Difference tests (HSDs) $P<0.001$ for both post hoc comparisons; psychological QoL: $F(2,538)=20.8, P<0.001$; Tukey's HSDs $P<0.01$ for both post hoc comparisons). There were no significant differences on disability or psychological QoL between individuals qualifying for a diagnosis of PTSD using the two-factor criteria only compared with those who met the three-factor criteria as well (Tukey's HSDs $P=0.933$ and $P=0.591$, respectively). Of note, no individual qualifying for a diagnosis of PTSD using the twofactor criteria only was rated as having a low level of disability (this was not the case for individuals meeting the three-factor criteria). Similarly, individuals who qualified for a diagnosis of PTSD using the three-factor criteria were no more likely to be identified as having poor psychological QoL than individuals who qualified for a diagnosis using the two-factor criteria only $\left(\chi^{2}=0.06, P=0.81\right)$.

Chi-squared tests of independence demonstrated no difference between individuals meeting the two-factor criteria only compared with those meeting the three-factor criteria in rates of comorbid major depressive episode, generalised anxiety disorder, panic disorder and travel phobia (major depressive episode: $\chi^{2}=1.0, P=1.0$; generalised anxiety disorder: $\chi^{2}=0.94, P=0.33$; panic disorder: $\chi^{2}=0.38, P=0.33$; travel phobia: $\chi^{2}=0.2 .4$, $P=0.12)$.

\section{Discussion}

The ICD-11 working groups have aimed to improve the clinical utility of diagnoses and their applicability across a range of socioeconomic and geographic contexts. For PTSD in particular, the working group has attempted to identify symptoms specific to the disorder and exclude symptoms shared with other conditions. ${ }^{1}$ As such, the ICD-11 re-experiencing symptoms of nightmares and flashbacks require that the event is not only remembered but experienced as occurring again' ${ }^{1}$ and thus exclude less specific forms of vivid memories and distress at reminders of the event, which have been shown to occur in a range of other disorders. ${ }^{37}$ Similarly, the ICD-11 hyperarousal symptoms no longer include irritability and sleep and concentration problems, which also occur in major depressive disorder and generalised anxiety disorder. ${ }^{38,39}$ Additional changes to the ICD-10 criteria for PTSD include requirements that the symptoms persist for at least several weeks and cause functional impairment. ${ }^{1}$

\section{Main findings}

Prior to our study, the latent structure of either ICD-10 or ICD-11 PTSD symptoms had not been investigated. ${ }^{40}$ Using CFA, the current study found that the three-factor model implied by the ICD-11 diagnostic criteria provided a good fit to symptom data drawn from injury survivors. However, the re-experiencing and avoidance factors were very highly correlated, and a more parsimonious two-factor model in which these factors were combined into a single factor provided an equivalent, and arguably superior, level of fit.

\section{Comparison with findings relating to DSM-5}

Our findings are consistent with recent studies, ${ }^{5,6}$ examining the latent structure of DSM-5 PTSD symptoms. ${ }^{4}$ The DSM-5 diagnostic criteria for PTSD imply a four-factor model, comprising re-experiencing, avoidance, negative alterations in cognition and mood, and arousal factors. Elhai et al, ${ }^{5}$ using self-report data drawn from a sample of college students exposed to a range of

Table 3 Mean disability and psychological quality of life scores ${ }^{a}$

\begin{tabular}{|c|c|c|}
\hline & \multicolumn{2}{|c|}{ Mean (s.d.) } \\
\hline & Disability & Psychological quality of life \\
\hline Individuals meeting two- or three-factor (ICD-11) criteria for PTSD & $15.9(8.3)$ & 46. $3(18.9)$ \\
\hline Individuals meeting two-factor criteria for PTSD only & $16.9(6.8)$ & $51.3(15.0)$ \\
\hline Individuals meeting neither criteria for PTSD & $6.4(7.2)$ & $64.1(12.3)$ \\
\hline \multicolumn{3}{|c|}{$\begin{array}{l}\text { PTSD, post-traumatic stress disorder. } \\
\text { a. Disability measured with the World Health Organization Disability Assessment Schedule } 2.0 \text { and psychological quality of life measured with the World Health Organization Quality } \\
\text { of Life - Bref psychological domain. }\end{array}$} \\
\hline
\end{tabular}


traumatic events, found the four-factor model implied by the DSM-5 diagnostic criteria demonstrated superior or equivalent fit to the other models tested. Miller et $a b^{6}$ using self-report data drawn from a representative sample of adults who met lifetime criteria for DSM-5 PTSD, found that an alternative four-factor model (including the same re-experiencing and avoidance factors) demonstrated superior fit to that implied by the DSM-5 diagnostic criteria. However, in both of these studies, the correlation between re-experiencing and avoidance was very high (0.95 in Miller et $a l^{6}$ and 0.89 in Elhai et $\left.a l^{5}\right)$, suggesting a lack of discriminant validity for these two factors.

\section{The case for a two-factor model}

Our study consequently investigated the impact of adopting diagnostic criteria based on a two-factor model combining the re-experiencing and avoidance factors on PTSD point prevalence. Adopting such criteria (at least two of: (a) nightmares, (b) flashbacks, (c) avoidance of thoughts or memories associated with the event, or (d) avoidance of people, places, conversations, activities or situations associated with the event; and at least one of hypervigilance or exaggerated startle response) resulted in a slight increase in the current prevalence of PTSD compared with the original ICD-11 criteria $(5.1 \%$ v. $3.4 \%)$. Using the same data as the current study, O'Donnell et al, found that the point prevalence of PTSD dropped from $8.8 \%$ for ICD-10 to $3.4 \%$ for ICD-11, largely because of the reduced number of possible re-experiencing and hyperarousal symptoms, although the ICD-11 requirement of functional impairment also contributed to this decrease. ${ }^{36}$ As the exclusion of non-PTSD-specific symptoms in the ICD-11 diagnostic criteria might be expected to result in a reduced prevalence rate, the slight increase in point prevalence produced by the criteria based on the two-factor model might be considered problematic. However, it may be that the proposed ICD-11 criteria are too restrictive to adequately capture trauma survivors experiencing significant distress. As suggested by O'Donnell et al, ${ }^{36}$ the re-experiencing component of the ICD-11 criteria may benefit from broadening to encompass distressing memories in addition to more literal ways of re-experiencing the traumatic event.

A critical function of a diagnostic cut-off is that it offers some differentiation between individuals with and without the disorder in terms of their burden of disability and QoL. In this study we found that using a more inclusive diagnostic algorithm for the proposed criteria based on the two-factor model of ICD-11 PTSD symptoms identified a group of individuals with similar levels of disability and poor psychological QoL compared with those identified using the proposed tripartite diagnostic criteria. This provides strong additional support for adopting the more inclusive criteria based on the two-factor model, as it suggests that the tripartite criteria fail to identify trauma survivors with similar symptom profiles and analogous functional impairment as having PTSD. This more inclusive diagnostic algorithm may offer an option for the WHO ICD-11 working group to ensure those with comparably compromised functioning and QoL are still identified using the proposed six PTSD specific symptoms.

The adoption of the two-factor model would enable individuals with either two re-experiencing symptoms or two avoidance symptoms in addition to at least one hyperarousal symptom to qualify for a diagnosis of PTSD. Such simplified criteria are consistent with early models of PTSD, which considered re-experiencing and avoidance symptoms as reciprocally related, or as 'two sides of the same coin. . $^{4-43}$

Given the intimate reciprocal relationship between reexperiencing and avoidance, the requirement for both in PTSD diagnostic algorithms can complicate assessment and diagnostic decisions for clinicians. Clinicians commonly assess traumaaffected patients for whom effortful avoidance (emotional, behavioural or substance-assisted) has been sufficiently effective to reduce re-experiencing symptoms to subthreshold levels or for whom highly distressing re-experiencing phenomena are tolerated with stoic determination to minimise avoidance. Such patients would not qualify for a diagnosis according to the tripartite ICD-11 criteria. However, the diagnostic algorithm indicated by the data from this study, in which two of any of the four re-experiencing and avoidance symptoms are sufficient, removes the requirement that decisions be made by clinicians on the basis of technicality rather than clinical severity in these cases. This is important as these decisions have implications for subsequent treatment.

This study has established that a diagnosis of ICD-11 PTSD based on the more inclusive two-factor diagnostic criteria is associated with similar levels of disability and reduced psychological QoL to a diagnosis based on the proposed tripartite criteria. Going one step further, however, this study also sought to ensure that the two-factor diagnostic criteria were not merely identifying additional individuals for whom equivalent disability and QoL scores might be attributable to higher rates of comorbid conditions. Individuals meeting the two-factor criteria only did not have higher rates of comorbid major depressive episode, generalised anxiety disorder, panic disorder and travel phobia than those individuals meeting the three-factor ICD-11 criteria for PTSD. This suggests that the levels of disability and poor psychological QoL in the former group cannot simply be attributed to the presence of comorbid diagnoses.

\section{Limitations}

The current study has some limitations that may affect the generalisability of its findings. First, as individuals in this sample had experienced significant physical injuries, other variables such as physical disability and pain may have contributed to disability and psychological QoL scores. Second, this study used the CAPS, a structured clinical interview to assess DSM-IV PTSD symptoms, instead of a measure designed to assess ICD-11 PTSD symptoms as they are specifically formulated. However, it should be noted that as the ICD-11 diagnostic criteria for PTSD are still at the proposal stage a validated measure of same is currently lacking. Third, this study examined ICD-11 PTSD symptoms 6 years post-injury, that is in the chronic phase. It may be that the latent structure of PTSD symptoms is different in more acute samples. In the DSM-5 literature, a number of other potential moderators of the latent structure of PTSD symptoms have been proposed (see Elhai \& Palmieri ${ }^{44}$ for a review), but we were unable to assess the impact of these in the present study. Further studies of the latent structure of ICD-11 PTSD symptoms in a range of samples taking into account potential moderating variables are required to establish the applicability of models to a broad range of contexts before considering their convergent and discriminant validity.

\section{Implications}

This study addresses, in a rigorous fashion, an issue that has thus far been neglected in the ICD PTSD literature. Whereas the threefactor model implied by the ICD-11 diagnostic criteria provided good fit to the data, a two-factor model provided an equivalent and arguably better level of fit. Although diagnostic criteria based on the two-factor model resulted in a slight increase in PTSD point prevalence, they identified a group of individuals with similar levels of disability and poor psychological QoL to the 
group who met criteria under the proposed tripartite PTSD model. The two-factor model supports the conceptualisation of re-experiencing and avoidance as intrinsically linked, consistent with early theoretical models of PTSD symptomatology. The findings also provide important information for the WHO ICD-11 working group regarding potential adjustments to the proposed ICD-11 PTSD criteria and its diagnostic algorithm to avoid exclusion of individuals with equivalent disability not accounted for by other disorders. The findings of this paper suggest that, in addition to the point raised by O'Donnell et al ${ }^{36}$ to broaden the proposed re-experiencing criteria, a more inclusive diagnostic algorithm based on the two-factor model may also address this need. However, confirmation of the goodness of fit of this model in a range of populations is required, as well as further examination of the potential discriminant validity of the re-experiencing and avoidance factors.

David Forbes, PhD, Emma Lockwood, PGDipPsych, Mark Creamer, PhD Australian Centre For Posttraumatic Mental Health and Department of Psychiatry, University of Melbourne, Victoria; Richard A. Bryant, PhD, School of Psychology, University of New South Wales, New South Wales; Alexander C. McFarlane, MD, Centre for Traumatic Stress Studies, University of Adelaide, South Australia; Derrick Silove, MD, School of Psychiatry, University of New South Wales, New South Wales: Angela Nickerson, PhD, School of Psychology, University of New South Wales, New South Wales; Meaghan O'Donnell, PhD, Australian Centre for Posttraumatic Mental Health and Department of Psychiatry, University of Melbourne, Victoria, Australia

Correspondence: David Forbes, Australian Centre for Posttraumatic Mental Health, Level 3/161 Barry St, Carlton, Victoria 3053, Australia. Email: dforbes@unimelb.edu.au

First received 16 Apr 2014, final revision 19 Jun 2014, accepted 6 Aug 2014

\section{Funding}

This study was supported by an Australian National Health and Medical Research Council (NHMRC) Program Grant (568970).

\section{References}

1 Maercker A, Brewin CR, Bryant RA, Cloitre M, Reed GM, van Ommeren M, et al. Proposals for mental disorders specifically associated with stress in the International Classification of Diseases-11. Lancet 2013; 381: 1683-5.

2 Brewin C. World Health Organization Preparation for ICD-11: Clinical Utility of Diagnostic Criteria for Trauma Related Disorders, Part 1 - Diagnosing PTSD from three core elements. Presented at 28th Annual Meeting of the International Society for Traumatic Stress Studies, 1-3 November 2012 Los Angeles, USA (http://www.istss.org/Expert_Training_ISTSS_Conferences/ 5920.htm).

3 Floyd FJ, Widaman KF. Factor analysis in the development and refinement of clinical assessment instruments. Psychol Assess 1995; 7: 286-99.

4 American Psychiatric Association. Diagnostic and Statistical Manual of Mental Disorders (5th edn) (DSM-5). APA, 2013

5 Elhai J, Miller ME, Ford JD, Biehn TL, Palmieri PA, Frueh BC. Posttraumatic stress disorder in DSM-5: estimates of prevalence and symptom structure in a nonclinical sample of college students. J Anxiety Disord 2012; 26: 58-64.

6 Miller MW, Wolf EJ, Kilpatrick D, Resnick H, Holowka BP, Keane DW, et al. The prevalence and latent structure of proposed DSM-5 posttraumatic stress disorder symptoms in U.S. national and veteran samples. Psychol Trauma 2012; 5: 501-12

7 Brown T. Confirmatory Factor Analysis. Guilford Press, 2006.

8 Kline RB. Principles and Practice of Structural Equation Modelling (3rd edn). Guilford Press, 2010.

9 Bryant RA, O'Donnell ML, Creamer M, McFarlane AC, Clark CR, Silove D. The psychiatric sequelae of traumatic injury. Am J Psychiatry 2010; 167 312-20

10 American Congress of Rehabilitation Medicine. Definition of mild traumatic brain injury. J Head Trauma Rehabil 1993; 8: 86-7.

11 Baker SP, O'Neil B, Haddon W, Long WB. The injury severity score: a method for describing patients with multiple injuries and evaluating emergency care. J Trauma 1974; 14: 187-96.
12 Blake DD, Weathers F, Nagy LM, Kaloupek DG, Gusman FD, Charney DS, et al. The development of a clinician administered PTSD scale. J Trauma Stress 1995; 8: 75-90.

13 Weathers FW, Keane TM, Davidson J. Clinician-administered PTSD scale: a review of the first ten years of research. Depress Anxiety 2001; 13 132-56.

14 American Psychiatric Association. Diagnostic and Statistical Manual of Mental Disorders (4th edn) (DSM-IV). APA, 1994.

15 Sheehan DV, Lecrubier $Y$, Harnett-Sheehan $K$, Amorim $P$, Janavs J, Weiller $E_{\text {, }}$ et al. The Mini International Neuropsychiatric Interview (M.I.N.I.): the development and validation of a structured diagnostic psychiatric interview. J Clin Psychiatry 1998; 59 (suppl 20): 22-33.

16 World Health Organization. The ICD-10 Classification of Mental and Behavioural Disorders: Clinical Descriptions and Diagnostic Guidelines. WHO, 1992.

17 WHODAS Group. World Health Organization Disability Assessment Schedule II. WHO, 2000

18 Perini SJ, Slade T, Andrews G. Generic effectiveness measures: sensitivity to symptom change in anxiety disorders. J Affect Disord 2006; 90 : 123-30.

19 Andrews G, Kemp A, Sunderland M, Von Korff M, Ustun T. Normative data for the 12 item WHO disability assessment schedule 2.0. PLOS One 2009; 4: e8343.

20 WHOQOL Group. Development of the World Health Organization WHOQOL-BREF Quality of Life Assessment. Psychol Med 1998; 28: $551-8$.

21 WHOQOL Group. WHOQOL-BREF: Introduction, Administration, Scoring and Generic Version of the Assessment. World Health Organization, 1996.

22 Hawthorne G, Herrman H, Murphy B. Interpreting the WHOQOL-Brèf: preliminary population norms and effect sizes. Soc Indic Res 2006; 77 37-59.

23 Barker-Collo S, Theadom A, Ameratunga S, Jones K, Jones A, Starkey N, et al. Prevalence and predictors of post-traumatic stress disorder in adults one year following traumatic brain injury: a population-based study. Brain Impair 2013; 14: 425-35.

24 Flora DB, Curran PJ. An empirical evaluation of alternative methods of estimation for confirmatory factor analysis with ordinal data. Psychol Methods 2004; 9: 466-491.

25 Wirth RJ, Edwards MC. Item factor analysis: current approaches and future directions. Psychol Methods 2007; 12: 58-79.

26 Muthén LK, Muthén BO. Mplus User's Guide (7th edn). Muthén \& Muthén, 2012.

27 Bandalos D. Is parcelling really necessary? A comparison of results from item parcelling and categorical variable methodology. Struct Equ Modeling 2008; 15: 211-40.

28 Elhai J, Palmieri P, Biehn T, Frueh BC, Magruder K. Posttraumatic stress disorder's frequency and intensity ratings are associated with factor structure differences in military veterans. Psychol Assess 2010; 22: 723-8.

29 Cole D, Ciesla JA, Steiger JH. The insidious effects of failing to include design-driven correlated residuals in latent variable covariance structure analysis. Psychol Methods 2007; 12: 381-98.

30 Hu LT, Bentler PM. Fit indices in covariance structure modelling: sensitivity to underparameterized model misspecification. Psychol Methods 1998; 3: 424-53.

31 Steiger JH. EZPATH: Causal Modelling. SYSTAT, 1989.

32 Bentler PM. Comparative fit indexes in structural models. Psychol Bull 1990; 107: $238-46$.

33 Tucker LR, Lewis C. A reliability coefficient for maximum likelihood factor analysis. Psychometrika 1973; 38: 1-10.

34 Cheung G, Rensvold R. Evaluating goodness-of-fit indexes for testing measurement invariance. Struct Equ Modeling 2002; 9: 233-55.

35 Raftery AE. Bayesian model selection in social research. Sociol Methodol 1995; 25: 111-63.

36 O'Donnell ML, Alkemade N, Nickerson A, Creamer M, McFarlane AC, Silove $D$, et al. Impact of the diagnostic changes to post-traumatic stress disorder for DSM-5 and the proposed changes to ICD-11. Br J Psychiatry 2014; 205 $230-5$.

37 Brewin CR, Gregory JD, Lipton M, Burgess N. Intrusive images in psychological disorders: characteristics, neural mechanisms, and treatment implications. Psychol Rev 2010; 117: 210-32.

38 Brewin CR, Lanius RA, Novac A, Schnyder U, Galea S. Reformulating PTSD for DSM-V: life after criterion A. J Trauma Stress 2009; 22: 366-73. 
39 Spitzer RL, First MB, Wakefield JC. Saving PTSD from itself in DSM-V. J Anxiety Disord 2007; 21: 233-41.

40 Andrews G, Charney DS, Sirovatka PJ, Regier DA. Stress-induced and Fear Circuitry Disorders: Refining the Research Agenda for DSM-V. American Psychiatric Association, 2008

41 Creamer $\mathrm{M}$, Burgess $\mathrm{P}$, Pattison $\mathrm{P}$. Reaction to trauma: a cognitive processing model. J Abnorm Psychol 1992; 101: 452-59.
42 Foa EB, Steketee G, Rothbaum BO. Behavioral/cognitive conceptualizations of post-traumatic stress disorder. Behav Ther 1989; 20: 155-76.

43 Horowitz MJ. Stress Response Syndromes (2nd edn). Jason Aronson, 1986

44 Elhai J, Palmieri P. The factor structure of posttraumatic stress disorder: a literature update, critique of methodology, and agenda for future research. J Anxiety Disord 2011; 25: 849-54.

\section{0 years ago}

\section{Gertrude Parker: a life in an asylum}

\section{Joy Parker}

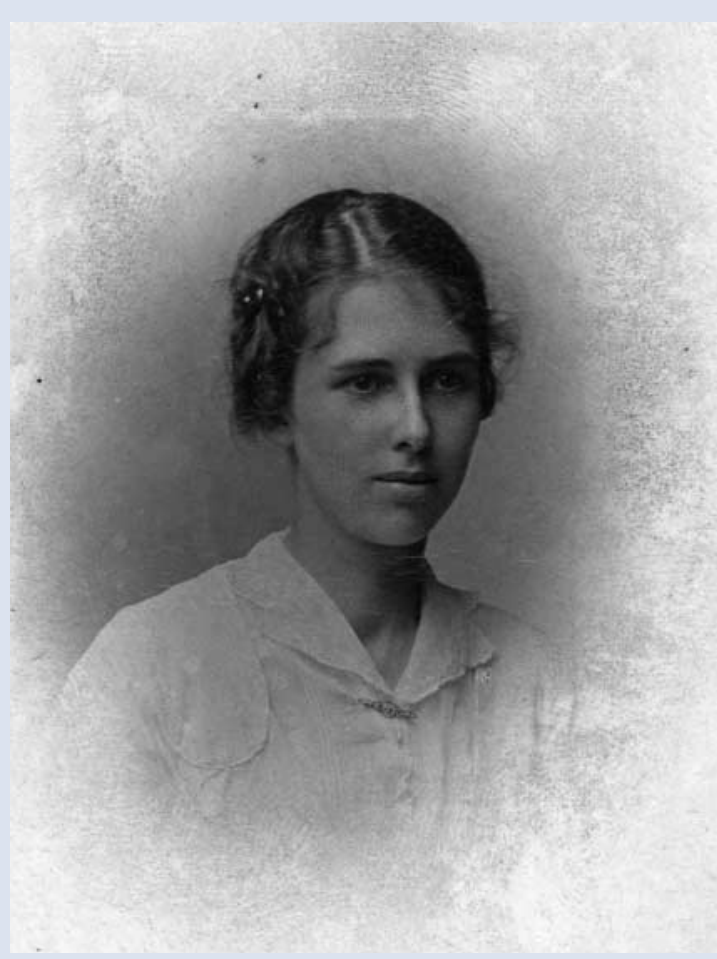

Gertrude Elline Parker was a long-term patient in Glenside Hospital, Bristol.

She was born in January 1898 in the village of Stoke-Sub-Hamdon in Somerset. In 1905, the family moved to a pleasant three-storey house in Bristol, where Gertrude grew up. She was artistically gifted: she painted and played the piano. In 1920, Gertrude married Edward Parker, a railway foreman on Avonmouth station. She was 22 and he 45. Initially, they lived in a disused army hut but they soon moved to Sea Mills, Bristol. Their first child, Raymond, was born in 1922. He was a sickly child and they did not know whether he would live, suspecting he might have tuberculosis. In 1924, Edward and Gertrude had a second son, Reginald.

It was at this point, when she had two young children, one of them critically ill in the children's hospital and the other only 11 months old, that Gertrude was first admitted to Bristol Lunatic Asylum, later known as Glenside Hospital. The admission record stated that she was suffering from chronic mania, caused by childbirth and prolonged stress. She was 27 years old.

Her case notes from this time record her behaviour as 'very restless, dishevelled and uncontrolled'. She was said to be '[talking] antics and [asking] conundrums ... . Often kneeling in a praying attitude and [saying] "I have blasphemed against the Holy Ghost".'

Perhaps her condition improved, because in 1928 she was discharged to Ashley Grange Care Home, Shirehampton. But after 6 years she returned to Glenside, where she spent the rest of her life.

While Gertrude was in Glenside, her husband visited her every other Sunday until his death in 1945. However, there is no record of her mother ever visiting, perhaps because of the social stigma attached to mental illness.

Gertrude's elder son Raymond, despite his ill health, lived to be a grown man. He married and adopted two children. Her younger son Reginald became a missionary in India, where he married an American, also a missionary. They spent 20 years in India and had five children. Reginald was not allowed to visit his mother until he was 16 years old, an experience he later described: 'When I visited my mother at the age of 16 she could not recognise me - whether she later had any understanding I do not know. Even though my grandmother and my aunt sought to "mother" me, I have never thought of anyone else as my mother. I look forward to meeting her in a more radiant form in another world.'

Gertrude Parker died in October 1969. On her death certificate the cause of death is recorded as 'bronchopneumonia and chronic schizophrenia'. 